\title{
A Spiral Contractometer for Measuring Stress in Electrodeposits
}

\author{
By Abner Brenner and Seymour Senderoff
}

\begin{abstract}
An improved instrument for the measurement of stress in electrodeposits is described. Its operation entails the electrodeposition of a metal coating on the outside of a metal helix and the measurement of the change in radius of curvature of the helix induced by the stress in the electrodeposit. The change in radius of curvature is read from a dial on the instrument. Formulas for the calculation of stress are given, and sources of error and correction factors for them discussed. Data are presented to demonstrate the reproducibility of measurements and the validity of the results. The variation of stress with plate thickness has been investigated and shown to be related to the variation in grain size of the deposit. The advantages of the instrument and fields for its application are indicated.
\end{abstract}

\section{Introduction}

It has been known for many years that frequently there is stress, either tensile or compressive, in electrodeposited metal coatings. This was discovered as early as 1877 by Mills [1] ${ }^{1}$, who called it "electrostriction." In 1909 Stoney [2] made the first quantitative measurements of stress in electroplates, using as the measuring instrument a straight metal strip, which was insulated on one side. Since then most investigators [3 to 11] in this field have used the same instrument, with certain refinements that will be noted later. The evaluation of stress by X-ray diffraction measurements has been attempted by a number of investigators [8, 12, 13, 14], but these results are difficult to interpret owing, among other factors, to the effect of grain size on X-ray patterns.

The main purpose of this paper is to describe an improved instrument for measuring stress in electrodeposits and to present formulas which may be used to calculate the stress. As there has been some confusion in the literature on the formulas for this calculation, a rigorous mathematical derivation and a discussion of the various formulas to show the correlation among them, and the condi-

\footnotetext{
1 Figures in brackets indicate the literature references at the end of this
} paper. tions of applicability of each, are presented in RP1954.

The second purpose of this paper is to demonstrate the effect of certain variables on the stress in electrodeposits.

Many authors have indicated the important effects of stress on the quality of plated articles. Excessively high stress may cause peeling, blistering, or cracking of the deposit and thereby render the base metal more susceptible to corrosion. It may also induce failures by the phenomenon of "stress corrosion."

It is desirable to use stress measurements not only in researches on plating, but also in production control to safeguard the quality of work. An instrument for such a purpose should be rugged, easily operated, self-contained, and comparatively inexpensive. The Stoney instrument and its modifications have proved valuable as research tools, but they do not adequately meet these other criteria.

Stoney [2], Phillips and Clifton [10], and Soderberg and Graham [11], placed a straight metal strip in a suitable fixture and plated the strip on one side only. Stoney permitted the strip to bend continuously during plating, whereas in the other two studies the strip was held rigid during plating and was allowed to bend some time after plating was stopped. In all three cases the radius of curva- 
ture of the bent strip was determined by measuring the camber (sagitta of the arc) of the bent strip. To do this, some type of special gage, such as was recommended by Phillips, or a microscope with a calibrated vernier focussing adjustment, is required.

Barklie and Davies [5], Marie and Thon [6], Jacquet [7], Hume-Rothery and Wyllie [8], and Martin [9], set the strip in a suitable fixture, allowed it to bend during plating, and observed and measured the displacement of one end of the strip through a microscope with a calibrated eyepiece. By this procedure, the displacement actually measured was four times that measured by Stoney's method for strips with equal radii of curvature.

Kohlschutter and Vuilleumier [3, 4], attached a thin glass pointer to the bottom of the strip at an acute angle, so that it emerged from the solution and passed across a scale when the strip bent during plating. This apparatus is fragile; it is difficult to attach the glass to the metal; and in addition, the sensitivity of the instrument is reduced by the weight of the glass at the end. The scale deflections observed are difficult to convert to absolute values of stress.

It will be noted that the first two methods require special auxiliary gages or calibrated microscopes for measurement and that the measured linear displacements are very small. The Kohlschutter apparatus is fragile, and it is difficult to determine the absolute values of stress from the observations made with it.

The new instrument developed at this Bureau meets to a reasonable degree the criteria for an instrument suitable for both production control and research. We have designated it the "spiral contractometer."

\section{Description and Operation of the Spiral Contractometer}

The spiral contractometer is based on the use of a helix, instead of a straight strip, for the measurement of stress. A flat strip is wound to form a helix and is plated on one side only. The stress in the deposit causes the helix to wind more tightly or to unwind, depending on whether the stress is compressive or tensile. When the plate is deposited under compression and relieves its stress by expanding, it exhibits "compressive stress." When the plate is deposited under tension and relieves its stress by contracting, it exhibits "tensile stress." The change in the radius of curvature of the helix is a measure of the stress in the plate. The change in radius of curvature is actually measured by the angular displacement of one end of the helix while the other end is held rigid. This angular displacement may be magnified conveniently by the use of gears and may then be read directly on the dial of the instrument. Figure 1, A, shows the assembled contractometer. Figure 1, B, shows the instrument with the helix detached. Figure 2 shows a modified instrument with the dial face vertical instead of horizontal. The parts of the instrument are shown in figure 3.

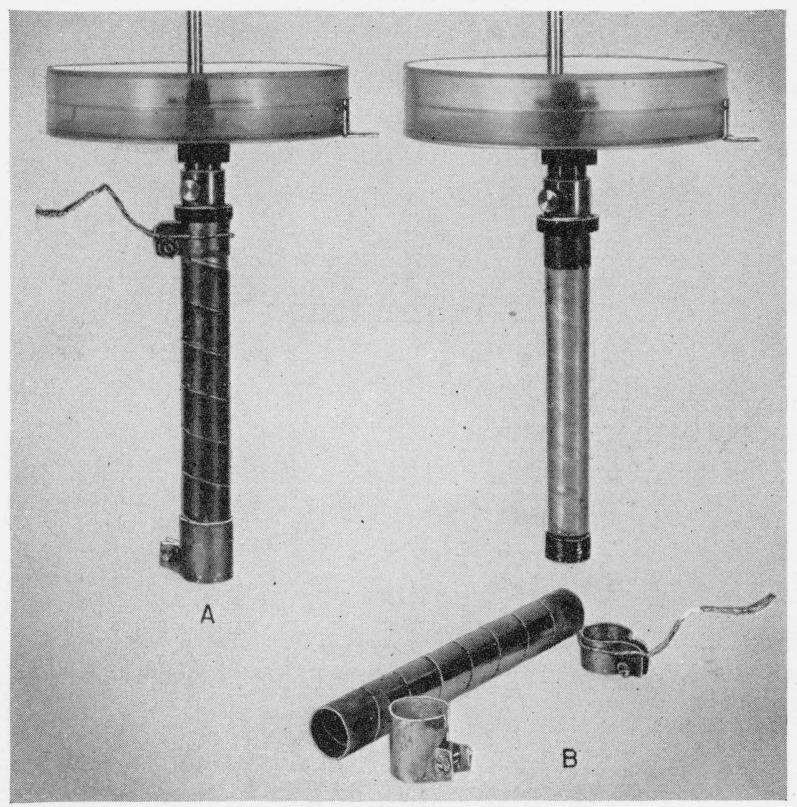

Figure 1. Spiral contractometer.

A, Contractometer completely assembled for stress determination; B, contractometer with helix removed.

The basic assembly (fig. 3) consists of a stainless steel disk, 7 , to the center of which is anchored a housing and bearings, 4 , for the pointer gear, 5 . The pointer, 2 , is rigidly anchored to its gear, which rotates freely with its shaft in the bearings. The entire pointer assembly is counterbalanced with a weight on the other side of the gear. One end of an arm, 3, is attached rigidly to the torque rod, 9. At the other end is a gear segment, 6, which meshes with the pointer gear. The pitch diameter of the gear on the arm is ten times as great as that of the pinion (or pointer gear) and gives tenfold magnification of the angular dis- 


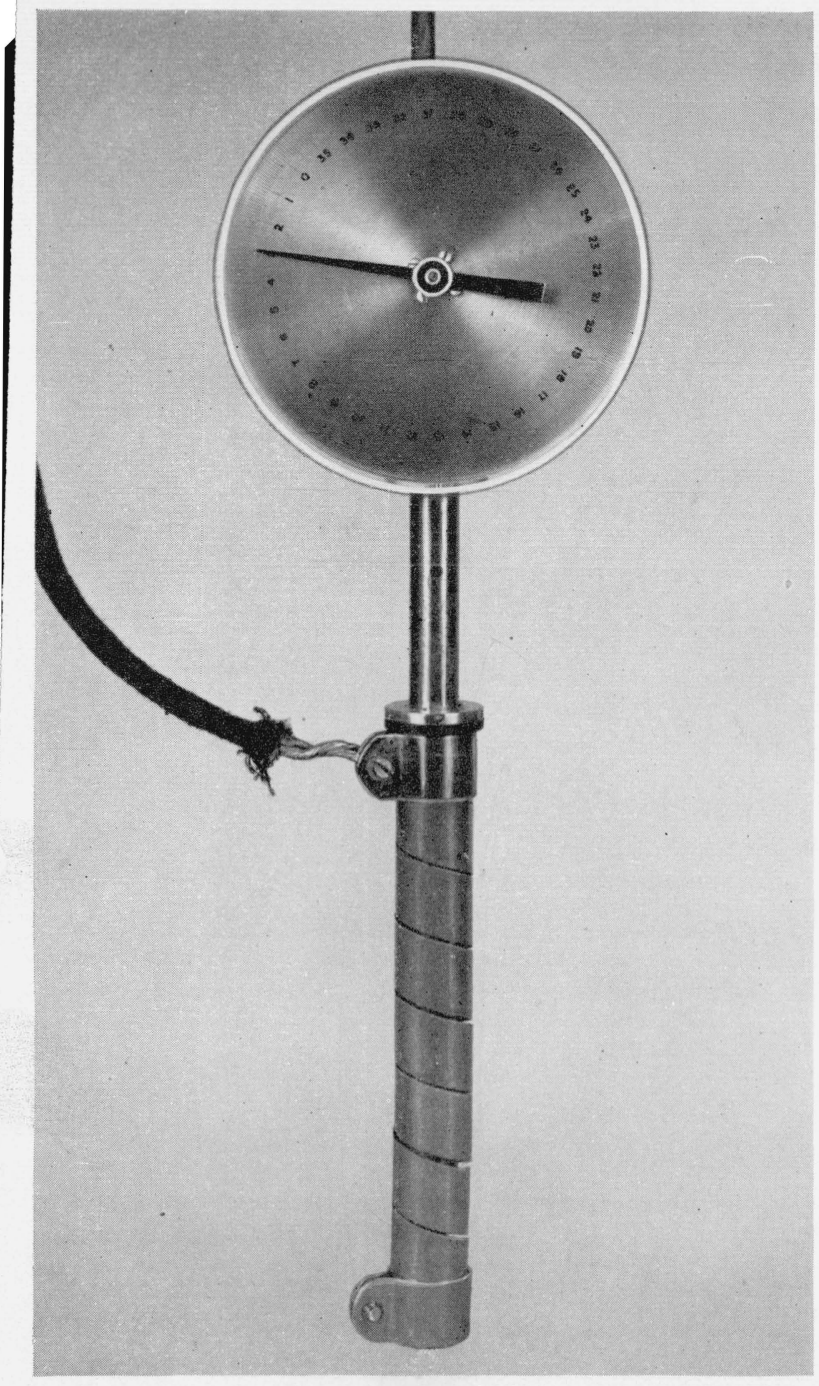

Figure 2. Model of contractometer with vertical dial face.

placement of the torque rod. The top end of the torque rod rotates freely in a small hole in the disk.

The guard tube (B) is slid on over the collar, 8 , and anchored with its set screw. The plastic base, 10 , is than attached to the torque rod with its countersunk set screw. The helix does not touch the guard tube. To attach it for a run, it is slid over the assembly and than locked in place, on the collar, 11, and the plastic base, 10, by clamps $D$ and $E$. These clamps serve not only to hold the helix tightly, but also, by acting as electrical guards, give a very uniform current distribution over the active length of the helix. By loosening the set screw on the guard tube $(B)$ the assembly is slid up or down over the collar,
8 , until the lever arm, 3 , is raised clear of the dial disk, 7, and the gears, 5 and 6 , properly mesh. By rotating the guard tube, the pointer may be set at any desired place on the dial. The set screw is then tightened, the plastic cap $(F)$ is placed on the instrument, and the entire assembly is suspended in a plating solution by the hanger, 1 , so that the solution level is somewhere on the top clamp $(D)$. By connecting the positive lead to a suitable anode and the negative lead to the wire on clamp $D$, plating on the helix proceeds. Since the top of the helix is firmly anchored, only the bottom turns during the plating. The rotation is transmitted to the pointer with a tenfold magnification and is read in degrees on the graduations at the edge of the disk.

Between normal operations the instrument need not be completely disassembled except for occasional cleaning. In that case, the disassembling and assembling can be done in less than $5 \mathrm{~min}$. For successive runs, only the helix and clamps need be removed or replaced, leaving the rest of the instrument assembled.

Since the helix is the heart of the instrument, it merits some detailed description. The helix is made by winding a metal strip of uniform width and thickness on a $3 / 4$-in. steel rod at a pitch such that there are neither spaces nor overlaps between the coils. The width of the strip is about $0.7 \mathrm{in}$. $(18 \mathrm{~mm})$. The thickness is determined by the rigidity desired for a particular application. The range of 0.01 to $0.03 \mathrm{in}$. ( 0.25 to $0.75 \mathrm{~mm}$ ) has been found most useful for this work. For deposits with very high stresses, thicker strips may be used. When the winding is completed, the ends are anchored to the rod by screws. The helix is then fully annealed in vacuo, in order to relieve the stresses due to cold working and thus prevent uncoiling. After annealing, the rod is mounted in a lathe and the helix is cut off at the desired length. The helix is then stretched lengthwise until the coils are separated from each other by a distance of about 0.02 to 0.04 in. (0.5 to 1.0 $\mathrm{mm})$. The inside rod is then removed and the helix is ready for use.

As there is some effort involved in properly preparing a helix and in measuring and calibrating it, it is fortunate that one helix can be used for many determinations. It was found that by using an 18:8 stainless steel helix for metal deposits that can be stripped with nitric acid, and a copper 


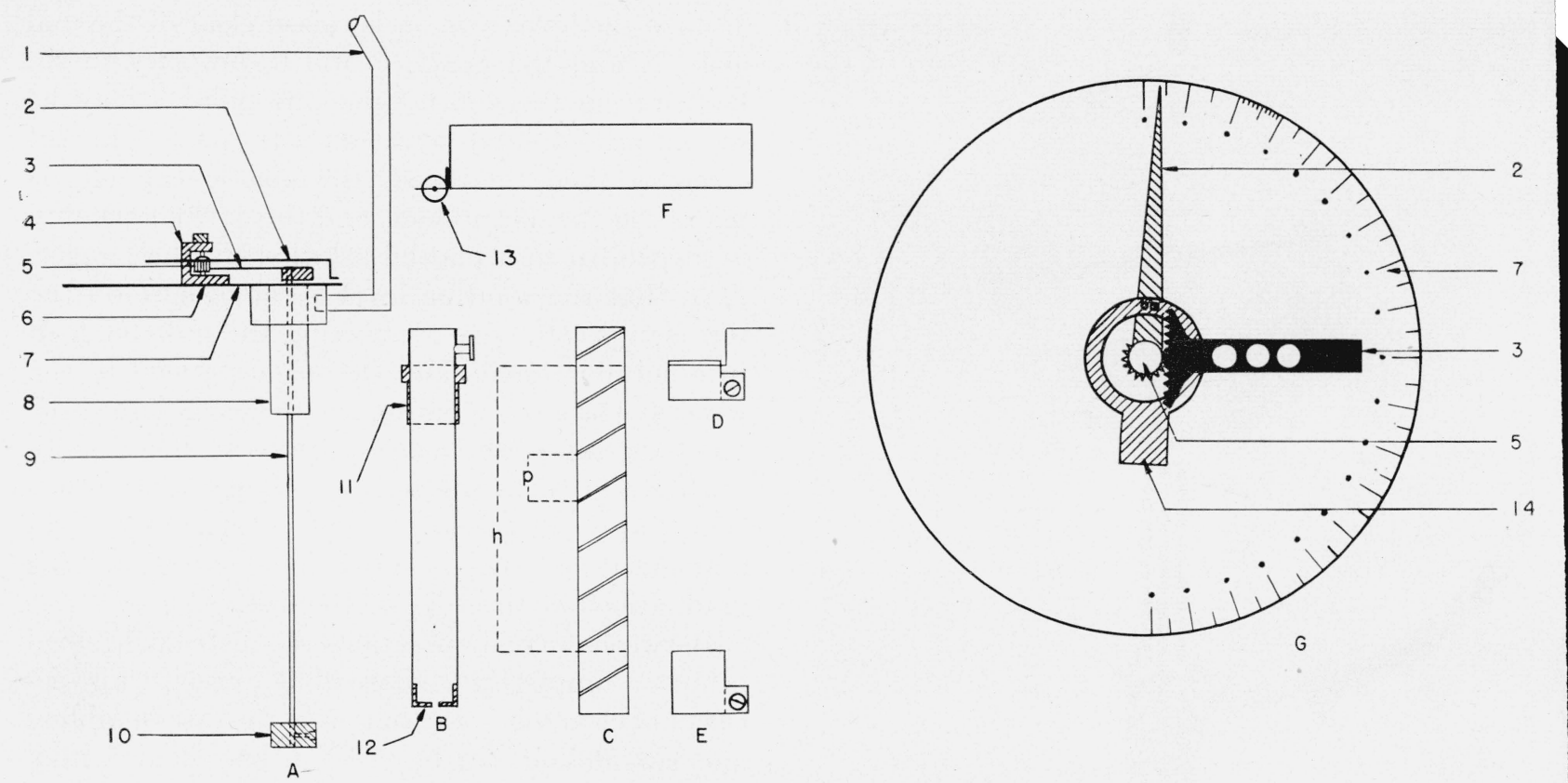

Figure 3. Schematic drawing of contractometer and its parts.

A, Basic assembly; B, guard tube; C, helix; D, top clamp with soldered wire lead; E, bottom clamp; F, plastic cap with removable calibration assemble; $\mathrm{G}$, top view of dial face. 1, hanger; 2, pointer; 3, lever arm; 4, housing and bearings; 5 , gear on pointer; 6, gear segment on lever arm; 7 , dial face graduated in degrees; 8 , collar; 9 , torque rod; 10 , plastic base with set screw; 11 , plastic collar; 12, plastic bearing; 13, removable pulley for ealibration; 14 , balancing weight on pointer.

helix for metals that can be stripped with hydrochloric acid, the helices can be reused almost indefinitely without appreciable change in their dimensions, elastic moduli, or deflection constants, provided reasonable precautions in handling and storing are used. When stainless steel helices are used, it is generally necessary to apply a special nickel strike, followed by a cyanide copper strike, to obtain good adherence of the deposit whose stress is being measured.

The steps involved in a stress determination are:

1. Clean the helix and apply a strike plate if desired.

2. Dry and weigh the helix.

3. Stop-off the inside of the helix by dipping a test-tube brush in thinned stop-off lacquer and passing it once through the helix.

4. Wipe the outside of the helix with acetone to remove the excess stop-off lacquer.

5. Dip in hot alkali and in an acid dip (or other suitable preplating dips) and rinse.

6. Mount the helix on the contractometer, set the clamps, adjust the pointer to the desired position, and replace the cap on the dial.
7. Immerse in the plating solution and start the plating.

The helix may be allowed to twist as plating progresses and a record kept of the displacements with time by taking periodic readings; or the pointer may be clamped before the plating starts and released at the end of the run. The clamping of the pointer prevents the helix from twisting until the plating is completed. Successive stress determinations by these two methods gave the same results within the experimental error.

8. At the end of a run, remove the helix, dissolve the lacquer from the inside of the helix with acetone, and clean and dry the helix.

9. Weigh the helix to determine the average thickness of the deposit.

10. Strip the deposit from the helix by suitable means.

The stress is calculated from the observed deflection, the physical constants and dimensions of the helix, and the average thickness of the coating, which is determined from the increase in weight of the helix.

For this calculation the following dimensions of 
the helix are measured with an accuracy of a few percent:

1. The outside diameter of the helix.

2. The thickness of the strip.

3. The pitch of the helix.

4. The height of the exposed portion of the helix between the clamps (or the plated portion) with the helix considered as a cylinder.

The first two are measured with a micrometer. The third and fourth quantities are determined by compressing the helix so that the coils just touch each other, and the appropriate distances measured with a centimeter rule. The height is $h$, and the pitch is $p$ in figure $3, \mathrm{C}$.

As will be shown later, the calculation is simplified if the deflection constant of the helix, that is, the torque required to cause a deflection of 1 degree is determined. This can be done by attaching a cotton thread to the lever arm near the gear segment, drawing the string through a hole in the side of the plastic cap and over the removable pulley, 13 (fig. 3) on the cap. With a helix clamped in place as described previously, weights are attached to the end of the string and the deflection is read on the dial. It is desirable to load the string with about 10 or $20 \mathrm{~g}$ as a zero load and to measure the deflection caused by increasing the load. The string is kept approximately perpendicular to the lever arm by rotating the cap in a plane parallel to the dial. By measuring the distance on the lever arm from the string to the torque rod, and using the known load in grams, a deflection constant that shows the relation of the bending moment to the degrees of deflection may be calculated for the helix.

\section{Calculation of Stress}

Although on certain projects it may be sufficient to express the stress at any stage by a scale deflection, as some investigators have done, it is preferable to express the results in absolute values. The formulas by which this may be accomplished are based on those of Stoney, with certain modifications to adapt them for use with a helix instead of a straight strip.

The following symbols will be used throughout this section:

$S=$ stress.

$S_{T}=$ true (or corrected) stress.

$E=$ Young's modulus of basis metal strip.
$E_{c}=$ Young's modulus of coating.

$p=$ pitch of strip.

$t=$ thickness of basis strip.

$d=$ thickness of deposit.

$\Delta(1 / r)=$ change of curvature induced by deposit.

$h=$ height of plated portion of helix.

$D=$ angular deflection of helix in radians.

$\bar{D}=$ angular deflection of dial needle in degrees.

$M=$ bending moment.

$K=$ deflection constant of helix.

$C=$ outside diameter of helix.

The basic formula used in this work is:

$$
S=\frac{E t^{2}}{6 d} \times \Delta\left(\frac{1}{r}\right)
$$

The change in curvature induced by the deposit (the difference between the initial and final curvature of the helix) is:

$$
\Delta\left(\frac{1}{r}\right)=\frac{D p}{\pi C h} .
$$

Substituting in eq 1 and rearranging terms,

$$
S=\frac{E t^{2} p}{6 \pi C h} \times \frac{D}{d},
$$

with $E$ in pounds per square inch; $t, C, h, p$, and $d$ in inches; and $D$ in radians; $S$ is in pounds per square inch. $E, t, C, h$, and $p$ are constants of the helix; $d$ is determined from the weight of deposit; and $D$ from the deflection of the needle on the instrument.

The derivation of this formula entails the assumption that the thickness of the deposit is negligible compared to the thickness of the basis metal strip. When the thickness of the deposit is appreciable, it must be considered for accurate results and this is done in Stoney's second formula:

$$
S_{T}=\frac{E\left(t^{2}+t d\right)}{6 d} \Delta\left(\frac{1}{r}\right)
$$

Equation 4 may be written in terms of eq 1 and a correction factor thus:

$$
S_{T}=S\left(1+\frac{d}{t}\right)
$$

A final refinement of the formula is required in order to take into account the difference in modulus which may exist between the basis metal and 
the deposit. This is done with a fair degree of accuracy (though it is not mathematically exact), by

$$
S_{T}=S\left(1+\frac{E_{c}}{E} \times \frac{d}{t}\right)
$$

One of the important advantages of the spiral contractometer is the fact that it is possible to calibrate it and thus eliminate errors due to differences in the physical properties of the helix from the published values usually associated with the metal from which it is made. The deflection constant of the helix is evaluated by

$$
M=K \bar{D}
$$

where $M=$ bending moment=weight of applied load $X$ distance from fulcrum. The stress may be calculated from this deflection constant by

$$
S=\frac{2 K}{p t} \times \frac{\bar{D}}{d}
$$

If $K$ is in inch-pound per degree, $\bar{D}$ in degrees, $p, t$, and $d$ in inches, then $S$ is in pounds per square inch. $S_{T}$ is obtained from $S$ by eq 6 , as indicated previously.

From the data obtained in calibrating a helix, its modulus can be calculated by

$$
E=\frac{2.16 \times 10^{4} K C h}{p^{2} t^{3}}
$$

where $K$ is known from the calibration, and $p$, $t, C$, and $h$ are measured dimensions of the helix. The use of these formulas in a typical stress determination is demonstrated in the appendix, p. 103.

The detailed discussion and derivation of these formulas appears in RP1954, but a few comments are in order here. The value of stress obtained by using the simpler approximate formulas 3 and 8 , can be seen to approach the true stress $\left(S_{T}\right)$ as $d / t$ approaches zero. Even when $d / t$ is as high as 0.1 , the values differ by only 10 percent, which may be considered to be the magnitude of the experimental error in a measurement of this type. In the experimental work described in this paper, the largest $d / t$ ratio used was 0.05 , and many

\footnotetext{
2 This equation applies to an instrument with a gear ratio of $10: 1$. The general formula is$$
E=\frac{2.16 \times 10^{3} K C h}{p^{2} t^{3}} g
$$

where $g$ is the gear ratio.
}

experiments were made with $d / t$ ratios of 0.03 or less. Best practice requires that $d / t$ be kept as low as practicable because of the departure of the behavior of the helix from the simple flexure theory and the increase in certain other errors as the radius of curvature decreases. When, however, $d / t$ in a particular experiment happens to be large or if higher accuracy is desired, it is simple to apply the correction factor of eq 6 to obtain an exact value for stress when $E_{c} / E=1$. Even when the moduli of the basis metal and coating differ, the value of $S_{T}$ obtained from eq 6 will differ from that obtained by the far more complicated exact formula by only a few percent, at most.

Lastly, it should be noted that the formula presented by Soderberg and Graham [11], i. e.,

$$
S_{T}=\frac{E(t+d)^{3}}{6 d t} \times \Delta\left(\frac{1}{r}\right),
$$

while slightly different from eq 4 , does not actually conflict with it. As shown in RP1954, the slight difference between the two is a result of the different experimental conditions used for measuring stress. In the present case, the strip is allowed to bend during plating, and in their experiment, it was held rigidly during plating. These cases require somewhat different mathematical treatment. The magnitude of the differences between stresses obtained by using the two formulas varies with $d / t$ and is about 10 percent when $d / t=0.05$.

In summary it may be said that for most ordinary applications of the spiral contractometer stress may be simply calculated by using eq 3 , or better, by using eq 8 . For high accuracy when $d / t$ and $E_{c} / E$ are high, the value thus obtained may be multiplied by a simple correction factor, as in eq 6, to obtain values of stress differing from the exact value by only a small fraction of the experimental error usually attending a stress determination.

\section{Evaluation of the Instrument}

Some of the factors that may affect the precision or accuracy of the instrument were studied. The current distribution over the helix was investigated by determining microscopically the thickness of metal deposited over the length of the helix. Under the most unfavorable conditions, which involved plating on the helix when it was immersed in a large plating tank, the average deviation of the plate thickness on the helix was 
\pm 3 percent from the mean. A stop-off lacquer must be used on the interior of the helix to prevent plating there. If too thick a layer is used it may affect the deflection constant of the helix. With a lacquer coating weighing about $0.2 \mathrm{~g} / \mathrm{dm}^{2}$, the calibration constant of the helix was changed by about 15 percent. However, a lacquer coating of about $0.05 \mathrm{~g} / \mathrm{dm}^{2}$, (about $0.0002 \mathrm{in}$., or $0.005 \mathrm{~mm}$, thick), had no significant effect on the deflection constant of the helix, and afforded satisfactory protection to the inside. In some experiments, plating on the inside of the helix was prevented by making the guard tube cathodic to the helix, in order that the inner surface of the helix would be anodic. This method works fairly well with acid solutions of copper and nickel, but not with solutions having good throwing power, such as the cyanide copper solution. There is also some uncertainty in determining the actual current on the outside of the helix.

Data presented in table 1 show that within experimental error, the nature of the helix used had no effect on the measurement of stress. Helices of 18:8 stainless steel and of copper, each in several different thicknesses, gave about the same results.

TABLE 1. Reproducibility of measurements with copper and stainless steel helices of various thicknesses

\begin{tabular}{|c|c|c|c|c|c|c|c|}
\hline Run & Helix & Type & $t$ & $d$ & $S$ & $S_{T}$ & $\begin{array}{l}\text { Correction } \\
\text { factor } \\
\left(1+\frac{E c}{E} \times \frac{d}{t}\right)\end{array}$ \\
\hline $1 \ldots$ & D & $\mathrm{Cu}$ & $\begin{array}{l}\text { in. } \\
0.0197\end{array}$ & $\begin{array}{l}\left(\text { in. } \times 10^{-4}\right) \\
\quad 6.58\end{array}$ & $\begin{array}{l}\text { lb/in. } .^{2} \times 10^{3} \\
\quad 15.5\end{array}$ & $\begin{array}{l}l b / i n .{ }^{2} \times 10^{3} \\
\quad 16.5\end{array}$ & 1.06 \\
\hline $2 \ldots$ & $\mathrm{E}$ & $\mathrm{Cu}$ & .0224 & 6.07 & 15.5 & 16.3 & 1.05 \\
\hline $3 \ldots \ldots$ & $\mathrm{E}$ & $\mathrm{Cu} \ldots$ & .0224 & 6.36 & 14.5 & 15.3 & 1.05 \\
\hline $4 \ldots \ldots$ & $\mathrm{F}$ & $\mathrm{Cu}_{\ldots} \ldots$ & .0219 & 6.16 & 13.8 & 14.5 & 1.05 \\
\hline 5. & $\mathrm{~F}$ & $\mathrm{Cu}$ & .0219 & 6.10 & 17.4 & 18.3 & 1.05 \\
\hline $6 \ldots$ & G & $\mathrm{Cu} \ldots$ & .0228 & 5.98 & 18.1 & 19.0 & 1.05 \\
\hline $7 \ldots$ & G & $\mathrm{Cu}$ & .0228 & 5.98 & 13. 2 & 14.0 & 1.05 \\
\hline $8 \ldots$ & $\mathrm{H}$ & $\mathrm{Cu} \ldots$ & .0315 & 5. 58 & 14.8 & 15.4 & 1.04 \\
\hline $9 \ldots .$. & B & Stainless steel... & .0131 & 6.62 & 14.7 & 15.5 & 1.05 \\
\hline $10 \ldots$ & $\mathrm{B}$ & _..._do do..... & .0131 & 6.62 & 14.7 & 15.5 & 1.05 \\
\hline 11. & l & ...... do ... & .0196 & 6.20 & 15.8 & 16.5 & 1.04 \\
\hline $12 \ldots$ & $J$ & ...... do _... & .0333 & 6.26 & 16.5 & 16. 9 & 1.02 \\
\hline \multicolumn{5}{|l|}{ Average (all runs) } & $15.4 \pm 7.5 \%$ & 16. $2 \pm 7 \%$ & \\
\hline \multirow{2}{*}{\multicolumn{5}{|c|}{$\begin{array}{l}\text { A verage } \mathrm{Cu} \text { helices only (8 determinations) } \\
\text { A verage stainless steel helices only ( } 4 \text { determinations) }\end{array}$}} & $15.388 \pm 8 \%$ & $16.123 \pm 8.5 \%$ & \\
\hline & & & & 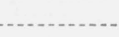 & $15.425 \pm 5 \%$ & 16. $100 \pm 3 \%$ & \\
\hline \multicolumn{5}{|c|}{ Percentage difference between averages for steel and $\mathrm{Cu}$ helices } & $0.2 \%$ & $0.2 \%$ & \\
\hline
\end{tabular}

Since the coating on a helix must be stripped after each run, the constancy of the calibration must be known. Stainless steel helix $B, 0.013$ in. $(0.33 \mathrm{~mm})$ thick was used during a period of 3 months for about 15 stress determinations. It was calibrated at various times, with the results shown in table 2. The constancy of the deflection constant, $K$, is clearly demonstrated, as well as the reproducibility of the calibration.

A source of error exists in the determination of the deflection constant of the helix by the direct loading method, if the helix is made from a thick metal strip. It was found that considerable torsion of the torque rod then occurred if large moments were applied to the lever arm (3 in fig. $3)$. On the other hand, no compensating error is
TABLE 2. Constancy of the calibration constant of stainless steel helices

\begin{tabular}{|c|c|c|}
\hline Elapsed time & $k$ & $K$ \\
\hline $\begin{array}{l}\text { Days } \\
0 \\
05 \\
40 \\
91\end{array}$ & $\begin{array}{l}\text { deg/g-cm } \\
2.13 \\
2.15 \\
2.14 \\
2.12\end{array}$ & $\begin{array}{l}\left.\quad \text { in. }-l_{b}\right) / d e g \\
4.06 \times 10^{-4} \\
4.03 \times 10^{-4} \\
4.05 \times 10^{-4} \\
4.10 \times 10^{-4}\end{array}$ \\
\hline Average (helix $B$ ). & $2.14 \pm 0.6 \%$ & $4.06 \times 10^{-4} \pm 0.6 \%$ \\
\hline
\end{tabular}

involved in measuring the stress in a coating, since only a negligible amount of torsion in the torque rod is required to turn the pointer. In a calibration, one can check whether or not the torsion in the torque rod is significant by keeping 
the bottom of the helix at rest when the turning moment is applied to the lever arm.

The reproducibility of the instrument was determined by making 12 stress determinations in a purified Watts nickel solution of the following composition :

\begin{tabular}{|c|c|c|c|}
\hline $\begin{array}{l}\mathrm{NiSO}_{4} \cdot 7 \mathrm{H}_{2} \mathrm{O} \ldots \ldots \\
\mathrm{NiCl}_{2} \cdot 6 \mathrm{H}_{2} \mathrm{O}_{\ldots} \ldots \\
\mathrm{H}_{3} \mathrm{BO}_{3} \ldots \ldots \ldots\end{array}$ & $\begin{array}{l}\quad{ }^{N} \\
\text { 1. } 8 \\
0.38 \\
.5(M)\end{array}$ & $\begin{array}{r}g / \text { liter } \\
240 \\
45 \\
30\end{array}$ & $\begin{array}{r}\text { oz/gal } \\
32 \\
6 \\
4\end{array}$ \\
\hline \multicolumn{4}{|c|}{$\mathrm{pH}=3.0$ to 3.5} \\
\hline
\end{tabular}

The determinations were made at $55^{\circ} \mathrm{C}$ and 2.5 $\mathrm{amp} / \mathrm{dm}^{2}\left(23 \mathrm{amp} / \mathrm{ft}^{2}\right)$, with approximately $30-\mathrm{min}$ runs, each giving approximately 0.0006 in. (0.015 $\mathrm{mm}$ ) of nickel deposit. Five copper helices and three stainless steel helices of varying thicknesses were used for the 12 runs. The copper helices were electrocleaned as cathode, dipped in a 20-percent $\mathrm{HCl}$ solution, rinsed and dried. After weighing them and stopping off the inside as described previously, they were wiped with acetone, dipped in the hot alkali cleaner, then in a 20 -percent $\mathrm{HCl}$ solution, and rinsed. They were then mounted on the instrument and plated. The stainless steel helices were electrocleaned anodically, dipped in 20 -percent $\mathrm{HCl}$, struck with nickel for $30 \mathrm{sec}$ at 6 $\mathrm{v}$ in a Woods nickel solution, rinsed, and struck with copper for $10 \mathrm{sec}$ at $4 \mathrm{v}$ in a cyanide copper solution. They were then rinsed, dried, and treated in the same way as the copper helices.

Table 2 shows the results of these runs. It can be seen that the average deviation from the mean in the series is less than \pm 8 percent, which may be considered satisfactory for a measurement of this type. A large part of these variations probably lies in the plating procedures rather than in the instrument. The average stress determined with the stainless steel helices differs by 1 percent from that obtained with the copper helices, which is well within the experimental error. The average deviation from the mean is less with the steel helices than with the copper. This may be caused by the greater dimensional stability of the steel helices and their smaller tendency to be permanently deformed on handling. For highest precision it seems advisable, therefore, to use stainless steel helices whenever applicable, that is, with any metal deposits that can be stripped with $\mathrm{HNO}_{3}$.
The validity of the results obtained with the instrument, that is, the extent to which the instrument measures stress and not some extraneous factors, cannot be directly determined, since there is no absolute method for measuring stress in deposits. The validity of the determinations of stress is confirmed by comparison with results obtained by other methods of measurement in baths of various types.

An indication of the validity of the calculations of stress was obtained by comparing the value of Young's modulus for a helix, as calculated from the deflection constant, with the known value. From the average value of $K$ for the stainless steel helix described in table 2, and eq 9 , the modulus is calculated to be $29.2 \times 10^{6} \mathrm{lb} / \mathrm{in}^{2}{ }^{3}{ }^{3}$ This compares with $29 \times 10^{6} \mathrm{lb} / \mathrm{in}^{2}$ for $E$ of $18: 8$ stainless steel as given in the literature [15]. A similar calculation of $E$ for the copper helices $\mathrm{D}-\mathrm{G}$ in table 1 gives an average value of $15.3 \times 10^{6} \mathrm{lb} / \mathrm{in}^{2}$ Timoshenko [16] lists $16 \times 10^{6}$ as $E$ for cold-rolled copper and Soderberg and Graham [11] report the value as $15.6 \times 10^{6}$.

It should be noted here that helices whose $k<$ approximately $0.75^{\circ} / \mathrm{g}$-cm give low values for $E$. This is explained by the fact that a portion of the bending moment applied in calibration is absorbed by the twisting of the torque rod of the instrument as indicated above. With the stiffer helices, such as $H, I$, and $J$, the portion absorbed by the torque rod becomes appreciable compared to the twisting of the helix, whereas with the weaker helices this portion is negligible. It is interesting to note, however, that a stress determination taken with a stiffer helix gives results equivalent to those obtained with the thinner helices, if the deflection observed with the stiff helices is used in eq 3 with the known modulus rather than in eq 8 with the calibration constant.

If it is necessary to consistently use stiff helices in some application, it would be desirable to use a thicker torque rod in order that the helices with lower values of $k$ could be calibrated.

In comparing values for stress in electrodeposits, the thickness of the deposit must be specified. The variation of stress with thickness will be considered later in some detail. Typical values for stress in nickel deposits plated from a carefully purified and electrolyzed Watts nickel

${ }^{3}$ This calculation is shown in detail in the appendix. 
solution of composition specified above, at $55^{\circ} \mathrm{C}$, $2.5 \mathrm{amp} / \mathrm{dm}^{2}$ and $\mathrm{pH} 3.0$ to 3.5 are shown in table 3 .

TABLE 3. Best values of stress in nickel deposited from Watts bath

Plating conditions: Watts nickel, temperature $=55^{\circ} \mathrm{C}, \mathrm{pH}=3.0$ to 3.5 , current density $=2.5 \mathrm{amp} / \mathrm{dm}^{2}$

\begin{tabular}{|c|c|c|}
\hline \multicolumn{2}{|c|}{ Stress } & \\
\cline { 1 - 1 }$S$ & $S_{T}$ & \\
\cline { 1 - 1 } & Thickness \\
\cline { 1 - 1 } lb/in.2 & lb/in. ${ }^{2}$ & \\
15,000 & 16,000 & Inch \\
12,000 & 13,000 & .0006 \\
9,000 & 11,000 & .001 \\
& & .002 \\
\hline
\end{tabular}

In the literature the published values for stress in nickel coatings from a Watts nickel solution vary from 13,000 to $25,000 \mathrm{lb} /$ in. $^{2}[17,5,10,11]$. As in most cases the thickness of plate, conditions of plating, or other pertinent data are not given, and as the stress varies with these factors and also with the purity of the plating solution, comparisons of table 3 with the published values are not of much value.

As all previous data on the stress in coatings, as well as those obtained with the helix, are based on the deformation of a metal strip, an attempt was made to measure stress by different procedures. A direct measurement of the contraction per unit length was made on a nickel shell deposited under stress and then freed of its base material. Brass tubes 6 in. $(15 \mathrm{~cm})$ long, $3 / 16$ in. $(0.5 \mathrm{~cm})$ in diameter, and $0.028 \mathrm{in} .(0.07 \mathrm{~cm})$ in wall thickness were plated with $0.002 \mathrm{in} .(0.05 \mathrm{~mm})$ of nickel from a Watts nickel solution. Shallow scratches were cut at intervals along the length of the tubes with a fine diamond tipped tool. The distances between these scratches were measured in a travelling microscope with an accuracy of better than \pm 0.0001 in. $(0.002 \mathrm{~mm})$. The brass was then removed by immersing the tube in a chromic and sulfuric acid mixture and bubbling air up through the tube. When the brass had completely dissolved, the distances between the scratches on the nickel tube were again measured. An average contraction in length of $3.0 \times 10^{-4}$ in./ in. was observed. By applying Hooke's Law and a small correction for circumferential and relieved stresses, a value of $10,300 \mathrm{lb} / \mathrm{in}^{2}{ }^{2}$ was obtained. Since the accuracy of measurement was about $\pm 0.0001 \mathrm{in}$. and the contraction in $4 \mathrm{in} .(10 \mathrm{~cm})$ (the greatest length measured) was about 0.0012 in. $(0.03 \mathrm{~mm})$, the accuracy of the measurement may be considered as approximately \pm 10 percent. As the accuracy of the measurements with the contractometer is also about \pm 10 percent, the agreement between the stress indicated for a 0.002 -in. $(0.05-\mathrm{mm})$ plate in table 3 , and this value may be considered fair.

Another instance of agreement with an independent method of measurement was secured in the following manner. A shell of chromium, approximately $0.005 \mathrm{in}$. thick $(0.13 \mathrm{~mm})$, was plated on a copper tube at $85^{\circ} \mathrm{C}, 80 \mathrm{amp} / \mathrm{dm}^{2}$. On dissolving the copper, the chromium developed a single longitudinal crack, having the full length of, and extending completely through the wall of, the shell. The amount by which the shell opened up at the crack is a measure of the internal stress in the chromium. The opening and the dimensions of the shell were measured with a microscope, and the average stress was calculated by the formulas given by Rosenthal and Mazia [18]. The average stress was found to be $53,200 \mathrm{lb} / \mathrm{in}^{2}$. Chromium was plated under similar conditions on the spiral contractometer, and a value of $60,000 \mathrm{lb} / \mathrm{in}^{2}$. was obtained. Again, the agreement may be considered satisfactory.

To more fully establish the validity of the determinations of stress made with the spiral contractometer, they were compared with the results of measurements made under similar conditions by the commonly accepted method of the bending of a flat strip. Simultaneous stress determinations were made with the spiral contractometer and a strip contractometer according to the method of Barklie and Davies [5]. Both instruments were used in the same solution at the same time. The results tabulated in table 4 show agreement of the two methods within 3 percent, which is within their experimental error.

TABLE 4. Comparison of spiral contractometer with the flat strip contractometer

\begin{tabular}{|c|c|c|c|c|c|c|}
\hline \multirow{2}{*}{ Run } & \multicolumn{3}{|c|}{ Spiral contractometer } & \multicolumn{3}{|c|}{ Strip contractometer } \\
\hline & $S$ & $S_{T}$ & $\left(1+\frac{d}{t}\right)$ & $S$ & $S_{T}$ & $\left(1+\frac{d}{t}\right)$ \\
\hline $\begin{array}{l}1 \ldots \\
2 \ldots\end{array}$ & $\begin{array}{c}l b / \text { in }^{2}{ }^{2} \\
15,000 \\
14,700\end{array}$ & $\begin{array}{c}l b / \text { in }^{2} \\
16,000 \\
15,600\end{array}$ & $\begin{array}{l}1.06 \\
1.06\end{array}$ & $\begin{array}{c}l b / \text { in }^{2} \\
16,000 \\
14,500\end{array}$ & $\begin{array}{c}l b / \text { in }^{2} \\
17,000 \\
15,400\end{array}$ & $\begin{array}{l}1.06 \\
1.06\end{array}$ \\
\hline Average ${ }^{1} \ldots$ & 14,900 & 15,800 & & 15,300 & 16,200 & \\
\hline
\end{tabular}

1 Difference in average $S=2.6 \%$. Difference in average $S_{T}=2.5 \%$. 
To study the response of the instrument to solution variants known to affect stress, the effects of impurities, high pH, chloride, hydrogen peroxide, and organic materials were investigated. The results shown in table 5 are in general agreement with the work of others $[3,9,10,11]$. There is some disagreement in the literature on the effect of hydrogen peroxide. The difficulty in measuring its effect may be associated with its decomposition with time. To check this effect further the strip contractometer was run simultaneously with the spiral contractometer in experiments 7 , 8 , and 9 (table 5), and the results are in excellent agreement.

TABLE 5. Effect of changes in bath composition on the stress in the nickel deposits

Thickness of deposit, $0.0006 \mathrm{in}$.

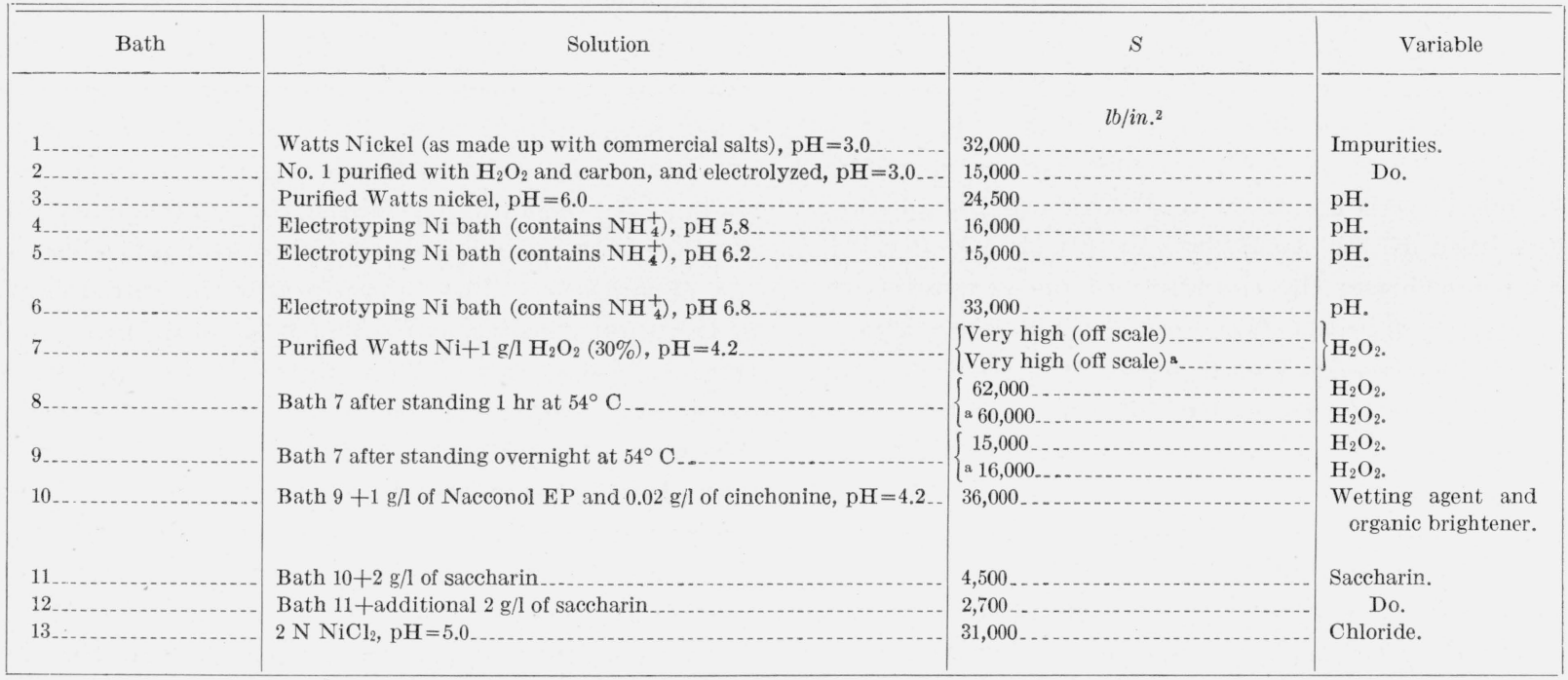

a Determination with straight-strip contractometer.

It has been observed that in runs made with either the spiral or strip contractometer, the stress usually decreased with thickness. This relation is shown in figures 4 and 5 . The same phenomenon was observed by Kohlschutter and Vuilleumier [3], and by Vuilleumier [4] in their work with the contractometer. They explained this on the basis of the progressive coarsening of the grain of the deposit with increasing thickness. Hughes, in commenting on Vuilleumier's paper suggested that the change might be due only to the stiffening of the strip, caused by increasing its thickness with the plating. That the effect is real and not due to increasing stiffness of the helix is shown by using the deflection constant for the plated helix in the calculation of stress. The increased stiffness of the helix accounts for only a small proportion $(10 \%)$ of the decrease in stress as the deposit increases from 0.0001 to 0.002 in. in thickness.

The stress which is determined for a coating of a given thickness is an average stress and is not a suitable quantity for studying the change of stress

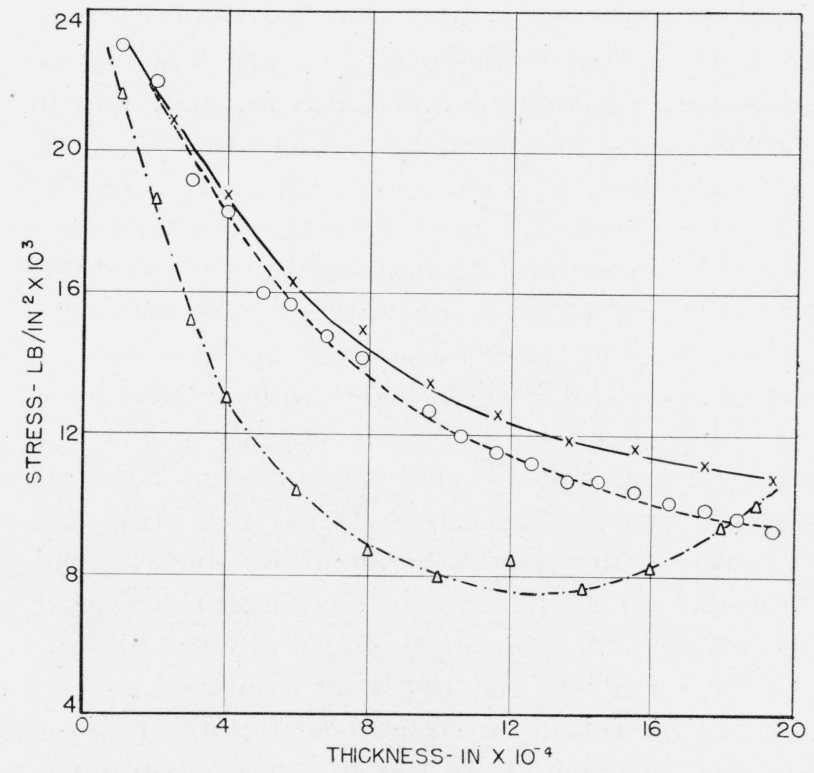

Figure 4. Stress in nickel, deposited from a carefully purified Watts nickel-plating solution.

......., Uncorrected stress $(S)$; —_ true stress $\left(S_{T}\right)$; ..._...., instantaneous stress $\left(S_{i}\right)$. 
with thickness of deposit. Suppose that a deposit had a high initial stress, which then decreased to a constant value as the coating became thicker, then the average stress would show a continual decrease with thickness which would mask the fact that the stress had become constant. Therefore, it is more sound to relate the thickness of the coating to the stress in the increment of coating deposited at that particular thickness. This stress will be referred to as the "instantaneous stress." It is readily determined from the slope of the curve for the average stress as shown in the appendix. In figures 4 and 5 the curves of the instantaneous stress are compared with the curves for the average stress. It will be seen that the instantaneous stress decreases rapidly until a thickness of about 0.0005 in. is reached.

The decrease of stress with thickness seems to be best explained on the basis of a change of grain size of the deposit. The following experiments were made to test this hypothesis.

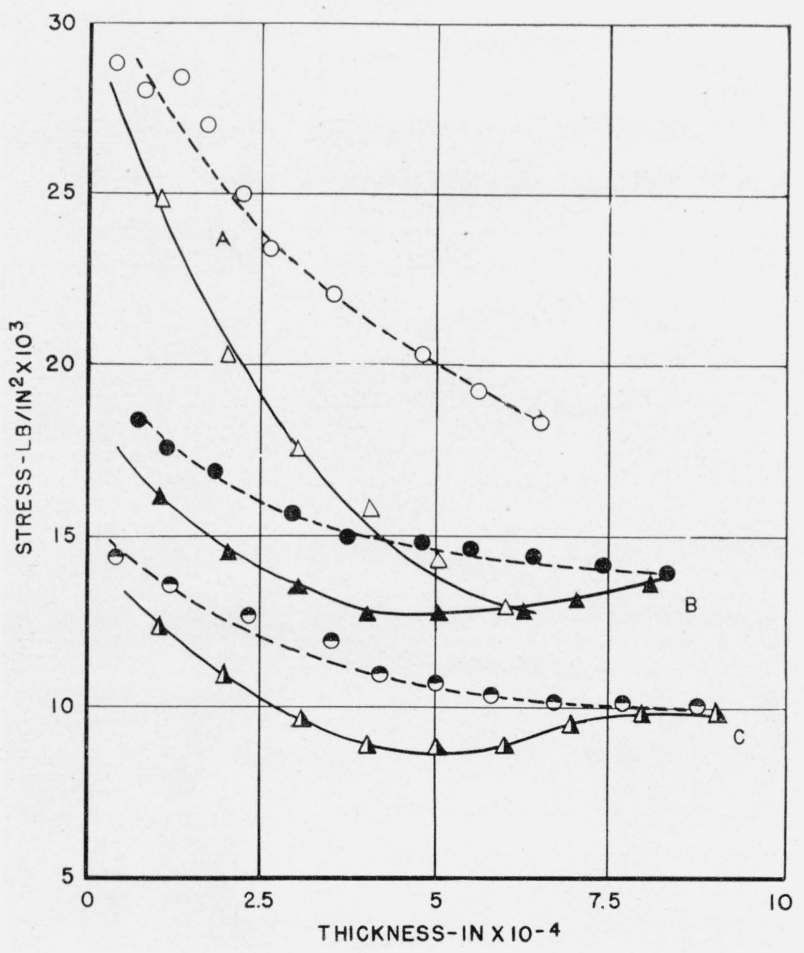

Figure 5.-Stress in nickel, deposited from pilot plant Watts nickel solution.

A, Stress of plate deposited on stainless steel helix; B, stress of plate deposited on bright (small grain) side of electroformed nickel helix; C, stress of plate deposited on dull (large grain) side of electroformed nickel helix; ........, true stress; ——, instantaneous stress.
A sheet of nickel approximately $0.014 \mathrm{in}$. $(0.34 \mathrm{~mm})$ thick was electroformed on a polished stainless steel base in a Watts nickel solution. The sheet was removed from the stainless steel base, and two longitudinal strips were cut from the center and formed into helices. One of the helices was wound so that the initially deposited bright surface of the nickel (the side originally adjacent to the stainless steel) was on the outside, and the other helix was wound so that the finally deposited dull surface (the side adjacent to the solution) was on the outside. These helices were annealed at $500^{\circ} \mathrm{C}$ for $1 \mathrm{hr}$ and were prepared for stress determinations as described previously. The helices were electrocleaned cathodically, activated by a 10 -sec cathodic treatment in 10percent $\mathrm{H}_{2} \mathrm{SO}_{4}$ and run in the nickel solution to a thickness of approximately $0.0008 \mathrm{in.}$ (The stress determination was run in the same solution in which the helices were electroformed.) The curves of figure 5 show the relation of thickness to stress found with the two helices. A curve for a run on a stainless steel helix in the same solution was included for comparison. It will be noted that the lowest stress was obtained when plating on the coarse-grained surface of the helix (curve c), the stress was higher when plated on the finegrained surface (curve b) and still higher when plating was done on the stainless steel helix (curve a). It will also be noted that the slopes of curves $\mathrm{b}$ and $\mathrm{c}$ are much smaller than that of curve a. An insight into this behavior can be obtained from inspection of the photomicrographs taken of cross sections of the two nickel helices with their subsequently applied deposits (fig. 6).

In each of the pictures the plate applied during the stress determination is on the right. In a and $\mathrm{c}$ the bright side of the helix is on the right, in b and $\mathrm{d}$, the dull side is on the right. In figure $6, \mathrm{a}$, and $6, \mathrm{~b}$, the progressive coarsening of the grain structure of the helix from the bright side toward the dull side can be seen. In figure $6, \mathrm{c}$, and $6, \mathrm{~d}$, it can be seen that the grain structure of the nickel plate in both cases is finer than that on which it was deposited, but the grain size of the plate in figure $6, d$, is larger than that in $6, c$, and there are more instances of continuance of the basis structure in 6 , d, than 6 , c. We may conclude, therefore, that the order of the stresses and the slopes of the curves shown in the curves of figure 5 are related to the grain size of the deposited nickel, and 

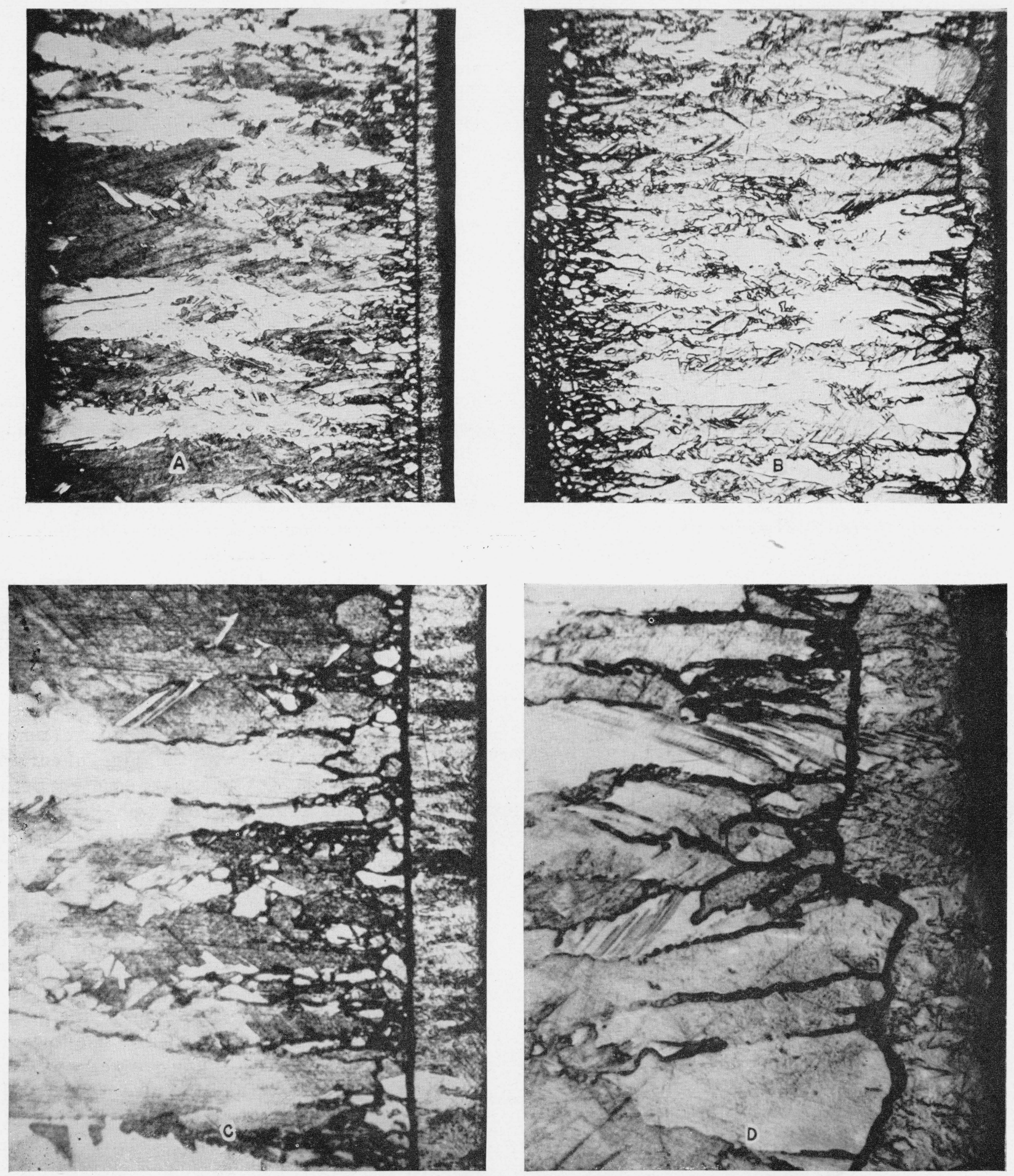

Figure 6.-Photomicrographs of cross sections of nickel plates on nickel helices.

In each case the nickel plate is on the right and the helix on the left. A, nickel plate on bright side of helix, $\times 150$; $\mathrm{B}$, nickel plate on dull side of helix, $\times 150$; $\mathrm{C}$, nickel plate on bright side of helix, $\times 500$; D, nickel plate on dull side of helix, $\times 500$. 
the amount of continuance of the basis structure. If there had not been some grain growth on the bright side of the helix (probably during annealing) curve 5 , b, would probably have approached 5, a, more closely, and if perfect continuance of the structure of the base nickel had been obtained, curve 5, c, probably would have more nearly approached a straight line. Close examination of figure 6 , a, and $6, \mathrm{~b}$, shows that the rate of grain growth of nickelobserved in the electroformed helix approximately corresponds to the rate of decrease of stress with increasing thickness shown in figure 4. It is rapid at first and then levels off. The low stress of certain bright nickel deposits, in spite of their very fine grain, does not detract from the plausibility of this analysis, because these plates invariably have a definite laminated structure and frequently contain impurities, such as compounds of sulfur or carbon. These extraneous factors may provide planes or areas for internal stress relief and introduce entirely new factors into the consideration of the mechanical forces in the deposit.

To determine whether the difference noted in curves 5 ,b and 5 ,c may be caused by the difference in micro-roughness of the surfaces of the two helices, a stress determination was run on a stainless steel helix, to a thickness of nickel of approximately 0.001 in. $(0.025 \mathrm{~mm})$. The contractometer was then removed from the solution and rinsed, and the deposit was electropolished to a mirror-bright surface in about $5 \mathrm{~min}$. After rinsing, the contractometer was returned to the nickel solution, and the stress determination was continued. A stress-thickness curve for the entire run duplicated figure 4 with only the slightest indication of any response to the electropolishing. As electropolishing smooths a surface without affecting the crystal structure, the measured stress must be related to the crystal structure and not to the micro-roughness of the surface.

From these tests it may be concluded that the stress in nickel deposits decreases with increasing grain size. The explanation of this is beyond the scope of this paper, but it may be related to inclusions at the grain boundaries as suggested by O'Sullivan [19], or possibly to the attraction between crystal faces as suggested by Blum and Rawdon [20]. The explanation of this phenomenon, together with the fundamental causes of stress in electrodeposits, is an important subject, on which further research is required.

\section{Application of the Spiral Contract- ometer}

The spiral contractometer may prove to be a valuable tool in the field of electrodeposition, both in research and in production control. A typical research application was the measurement of the stress of chromium deposits reported in the paper on properties of chromium by Brenner et al. [21]. In this study, the stress of chromium deposits produced at various temperatures was measured with the spiral contractometer. It was found that the high-temperature crack-free deposits (frequently referred to as "stress-free") actually were under high stress. The "low-stress" deposits produced in the vicinity of the "bright range" were low in stress as a result of stress-relief by cracking. It was shown that when they were very thin, these deposits also built up considerable stresses before they cracked. It was concluded that the high-temperature conditions produced chromium of higher tensile strength (probably because of its lower oxide content), so that the high stresses of the chromium deposits could exist without producing cracking. Figure 7 shows the results obtained with chromium, and in addition, the curve for nickel drawn on the same scale for comparison.



Figure 7. Stress in chromium plate deposited at various temperatures.

A curve for stress in nickel is included for comparison. 
Cracking of a deposit is not in itself an indication of the magnitude of stress, but rather it is an indication that the maximum stress at certain points exceeds the tensile strength of the material. Cracking can be induced either by conditions that increase the stress or lower the tensile strength. Inclusions, such as oxides, or basic salts, therefore, may not actually raise the stress, but they may lower the tensile strength of the deposit.

The spiral contractometer has been used to measure stresses of metals deposited from other solutions such as cobalt, cobalt-tungsten alloys; and "electroless nickel and cobalt" where metal deposition is accomplished by chemical reduction, without the use of current. This latter use suggests a possibility of its application for measuring stresses in nonmetallic or organic coatings that are applied by brushing or spraying.

Other research problems that might be aided by the spiral contractometer are the search for stress-reducing agents, the determination of plating conditions that give minimum stress, and the determination of the effects on stress of addition agents used for various purposes in plating solutions.

An important field of application of the spiral contractometer is in production control. By periodic stress determinations in an operated plating tank, the presence of contamination can be detected. Purifying treatments can be followed with the contractometer, and the effectiveness and completeness of the treatment determined. During production, periodic stress determinations can be made with this instrument while plating is done in the tank, and with current from the regular busbar. The instrument may also be valuable in "trouble-shooting", especially when the adhesion is to be improved. By a simple measurement, abnormal plating stresses may be detected, and thus either eliminated or confirmed as a cause of poor adhesion.

\section{Summary and Conclusions}

A new instrument for the measurement of stress in electrodeposits has been described. The spiral contractometer has been shown to be a rugged, easily operated unit that gives measurements of stress that are reproducible in nickel solutions to within \pm 10 percent. The values obtained with this instrument agree well with reported values, with measurements made with another type of contractometer, and with measurements by procedures based on different principles. The instrument is sensitive enough and accurate enough for research work. The convenience of its use should encourage its application to routine control of plating solutions and plated products. The advantages of the design of this instrument as compared to others are (1) Convenience: No auxiliary optical or other types of measuring equipment are required. The measurement of deflection is taken from a dial on the instrument. This permits the instrument to be hung in an operating plating tank and measurements to be taken on the site. (2) Sensitivity: The length of strip that may be conveniently used when wound as a helix is four to eight times that usually used in the straight-strip instruments. This gives a greater deflection for a given change in curvature. The deflection can be conveniently magnified to any desired degree by the gear system of the instrument. (3) The measurements are made at the plating temperature, thereby eliminating thermal errors. (4) The use of a coiled strip greatly reduces or eliminates errors caused by transverse bending. (5) The instrument can be easily calibrated, thus permitting simpler calculations and eliminating errors due to variation of the physical properties of the helix.

The values for stress in pure nickel deposits of various thicknesses and deposited under clearly defined conditions have been established.

A simple formula for the calculation of stress in electrodeposits has been presented and it has been shown to be sufficiently accurate for most work. Where special conditions require it, a correction factor for use with that formula has been derived, by which values of higher accuracy may be calculated without the use of complicated formulas requiring extended computations. In addition, another paper has been prepared for publication in which the formulas for calculating stress are rigorously derived and the relations of previously suggested formulas are shown.

The change in stress of nickel deposits with thickness has been investigated and shown to be related to the increase in grain size as plating progresses.

The authors acknowledge the cooperation and assistance of the staff of the Bureau's Electrodepo- 
sition Section, as well as members of other sections of the Bureau. In particular we acknowledge the contribution of $\mathrm{R}$. Crosthwaite, who assisted in the designing of the instrument, and that of $\mathrm{W}$. Ramberg and S. Levy of the Engineering Mechanics Section, who advised us on matters relating to the theory of elasticity.

\section{References}

[1] E. J. Mills, Proc. Roy. Soc. (London) 26, 504 (1877).

[2] G. G. Stoney, Proc. Roy. Soc. (London) A82, 172 (1909).

[3] V. Kohlschutter and E. Vuilleumier, Z. Electrochem. 24, 300 (1918).

[4] E. Vuilleumier, Trans. Electrochem. Soc. 42, 99 (1922).

[5] R. H. D. Barklie and H. J. Davies, Proc. Inst. Nech. Eng., p. 731 (1930), I.

[6] C. Marie and N. Thon, J. Chim. Phys. 29, 11 (1932).

[7] P. Jacquet, Compt. Rend. 194, 456 (1932).

[8] W. Hume-Rothery and M. R. Wyllie, Proc. Roy. Soc. (London) A181, 331 (1943); A182, 415 (1944).

[9] B. Martin, Proc. Am. Electroplaters' Soc., p. 206 (1944).

[10] W. Phillips and F. L. Clifton, Proc. Am. Electroplaters' Soc., p. 97 (1947).

[11] K. G. Soderberg and A. K. Graham, Proc. Am. Electroplaters' Soc., p. 74 (1947).

[12] W. A. Wood, Phil. Mag. 15, 553 (1933); 20, 964 (1935); 24, 772 (1937).

[13] E. M. Mahla, Trans. Electrochem. Soc. $7 \%, 145$ (1940).

[14] M. R. J. Wyllie, J. Chem. Phys. 16, No. 1, 52 (1948).

[15] Metals Handbook of Am. Soc. Metals, p. 541 (1939).

[16] S. Timoshenko, Strength of materials, part I, p. 5, 2nd ed. (D. Van Nostrand and Co., New York, N. Y., 1940).

[17] A. W. Hothersall, Proc. Am. Electroplaters' Soc., p. 4 (1946); J. Inst. Metals, Symposium on Internal Stress in Metals and Alloys, Preprint No. 1082 (1947).

[18] H. Rosenthal and J. Mazia, Symposium on Stress Corrosion Cracking of Metals, p. 122 (1944), Am. Soc. Testing Materials and Am. Inst. Mining Met. Engrs.

[19] J. B. O'Sullivan, Trans. Faraday Soc. 26, 89 (1930).

[20] W. Blum and H. S. Rawdon, Trans. Electrochem. Soc. 44, 397 (1923).

[21] A. Brenner, P. Burkhead, and C. W. Jennings, Proc. Am. Electroplaters' Soc., p. 32 (1947); J. Research NBS 40, 31 (1948) RP1854.

\section{Appendix}

Some calculations based on data taken with the spiral contractometer are demonstrated herein.

The calculation of stress of run 9 , table 1 , follows:

Dimensions of Helix (Helix B)

$C=$ outside diameter of helix $=0.765 \mathrm{in}$. $(1.94 \mathrm{~cm})$. $p=$ pitch of helix $=0.678 \mathrm{in} .(1.72 \mathrm{~cm})$.

$t=$ thickness of strip $=0.0131 \mathrm{in} .(0.33 \mathrm{~mm})$.

$h=$ height of plate $=4.49 \mathrm{in} .(11.4 \mathrm{~cm})$.

$k=5.57 / 2.60=2.14 \mathrm{deg} / \mathrm{g}-\mathrm{cm}$.

$K=4.05 \times 10^{-4}$ in.-lb/deg (deflection constant).

$h$ and $p$ are measured by mounting the plated helix on a $3 / 4-$ in. rod, compressing it longitudinally until the coils of the helix just touch each other and measuring the height of the deposit and the pitch of the helix with a centimeter rule.

$K$ is obtained as follows: In calibrating this helix it was found that the average deflection of the pointer for 0 - to 25 -g loads was $5.57 \mathrm{deg} / \mathrm{g}$ with the load attached to the lever arm at a distance of $2.60 \mathrm{~cm}$ from the torque rod, and $k=5.57 / 2.60=2.14 \mathrm{deg} / \mathrm{g}-\mathrm{cm}$.

$K=8.68 \times 10^{-4} / k=4.05 \times 10^{-4} \mathrm{in} .-\mathrm{lb} / \mathrm{deg}$ since $1 \mathrm{~g}$ - $\mathrm{cm}=$ $8.68 \times 10^{-4}$ in. $\left.-1 b\right)$.

The weight of nickel on the helix $=1.04 \mathrm{~g}$.

where,

$$
d=\frac{g}{G \times 2.54 \times \pi C h},
$$

$g=$ weight of plate in grams.

$G=$ density of nickel.

$C=$ diameter of helix in centimeters.

$h=$ height of plated portion of helix.

$d=$ thickness of plate in inches.

$$
d=\frac{1.04}{8.9 \times 2.54 \times 3.14 \times 1.94 \times 11.4}
$$

$d=1.04 / 1575=6.62 \times 10^{-4}$ in.

It will be noted that for a given helix and plated metal the denominator is constant. The plate thickness may therefore be obtained by merely dividing its weight by the constant denominator.

The pointer deflection, $D$, observed for this deposit was $107^{\circ}$.

Substituting values in eq 8

$$
\begin{aligned}
S= & \frac{2 K}{p t} \times \frac{\bar{D}}{d}=\frac{2 \times 4.05 \times 10^{-4}}{0.678 \times 1.31 \times 10^{-2}} \times \frac{107}{6.62 \times 10^{-4}}= \\
& 9.13 \times 10^{-2} \times 1.615 \times 10^{5}=14,700 \mathrm{lb} / \mathrm{in}^{2} .
\end{aligned}
$$

It will be noted here that the first term, $2 K / p t$, is constant for a given helix and that the stress is determined by multiplying the ratio, $\bar{D} / d$, by this constant.

To determine stress by use of the known $E$ of the helix instead of $K$, the observed deflection of the pointer in degrees, $\bar{D}$, is converted to deflection of the helix in radians, $D$.

$107^{\circ} / 10=10.7^{\circ}=$ deflection of helix, since the gear ratio is $10: 1$.

$10.7^{\circ} \times \pi / 180=1.87 \times 10^{-1}$ radians $=D$

Substituting in eq 3 ,

$$
\begin{gathered}
S=\frac{E t^{2} p}{6 \pi C h} \times \frac{D}{d}=\frac{29 \times 10^{6} \times 1.72 \times 10^{-4} \times 6.78 \times 10^{-1}}{6 \times 3.14 \times 7.65 \times 10^{-1} \times 4.49} \times \\
\frac{1.87 \times 10^{-1}}{6.62 \times 10^{-4}}=52.4 \times 282=14,800 \mathrm{lb} / \mathrm{in}^{2} .
\end{gathered}
$$


$E$ stainless steel, helix $\mathrm{B}=29 \times 10^{6} \mathrm{lb} / \mathrm{in}^{2}$.

Again the first term, $E t^{2} p / 6 \pi C h$, is constant for a given helix.

The correction factor to obtain the true stress, $S_{T}$, is applied according to eq 6 .

$$
\begin{aligned}
S_{T} & =S\left(1+E_{c} / E d / t\right) \\
& =14.7 \times 10^{3}(1.050) \\
& =15,500 \mathrm{lb} / \mathrm{in}^{2} .
\end{aligned}
$$

$$
\begin{aligned}
& E_{c} / E \text { for nickel on steel }=1 \\
& d / t=6.62 \times 10^{-4} / 1.31 \times 10^{-2} \\
& \quad=0.050
\end{aligned}
$$

The modulus of the helix is calculated from the calibration data as follows:

Table 2 gives $K=4.06 \times 10^{-4} \mathrm{in} .-\mathrm{lb} / \mathrm{deg}$ for helix B.

Substituting in eq 9

$$
\begin{aligned}
E= & 2.16 \times 10^{4} \mathrm{KCh} / \mathrm{p}^{2} t^{3} \\
= & 2.16 \times 10^{4} \times 4.06 \times 10^{-4} \times 7.65 \times 10^{-1} \times \\
& 4.49 / 4.60 \times 10^{-1} \times 2.25 \times 10^{-6} \\
= & 29.2 \times 10^{6} \mathrm{lb} / \mathrm{in}^{2}
\end{aligned}
$$

There is no need to calculate the modulus of a helix for a stress determination, but it serves as a good check on the physical condition of a helix.

\section{Calculation of Instantaneous Stress $\left(S_{i}\right)$}

At thickness $t, S_{a}=1 / t \int_{0}^{t} S_{i} a t$, where $S_{a}=$ stress (corrected) shown by the contractometer at thickness $t$.

$S_{a} t=\int_{0}^{t} S_{i} d t$; and differentiating with respect to $t$, $S_{a}+t d S_{a} / d t=S_{i}$

But since $d S_{a} / d t$ is the slope of the stress versus thickness curve, at thickness $t$,

$d S_{a} / d t=m_{l} / t$ and $S_{i}=S_{a}-m$ (see fig. 8).



Figure 8. Diagram showing calculation of instantaneous stress $\left(S_{i}\right)$.

The tangent of the $S_{T}$ curve in figure 5 at various thicknesses was found graphically, and its intercept, $y$, on the stress axis noted on the graph. Since $m=y-S_{a}$, and $S_{a}$ can be read from the curve, $S_{i}$ can be determined at any thickness.

Washington, June 18, 1948. 